\title{
Investigation of the Relationship between MR-Based Supraclavicular Fat Fraction and Thyroid Hormones
}

\author{
Daniela Junker ${ }^{a} \quad$ Jan Syväri ${ }^{a}$ Dominik Weidlich ${ }^{a} \quad$ Christina Holzapfel $^{b}$ \\ Theresa Drabsch $^{b}$ Birgit Waschulzik ${ }^{c}$ Ernst J. Rummeny ${ }^{a}$ \\ Hans Hauner $^{b}$ d Dimitrios C. Karampinos ${ }^{a}$ \\ ${ }^{a}$ Department of Diagnostic and Interventional Radiology, Klinikum rechts der Isar, School \\ of Medicine, Technical University of Munich, Munich, Germany; ${ }^{b}$ Institute for Nutritional \\ Medicine, Klinikum rechts der Isar, School of Medicine, Technical University of Munich, \\ Munich, Germany; ' Institute of Medical Informatics, Statistics and Epidemiology, Klinikum \\ rechts der Isar, School of Medicine, Technical University of Munich, Munich, Germany; \\ ${ }^{d}$ Else Kroener-Fresenius-Center of Nutritional Medicine, ZIEL Institute for Food and Health, \\ Technical University of Munich, Freising, Germany
}

\section{Keywords}

Thyroid hormones - Brown adipose tissue - Proton density fat fraction · Adults - Magnetic resonance imaging

\begin{abstract}
Purpose: Brown adipose tissue (BAT) plays a potential role in energy and glucose metabolism in humans. Thyroid hormones (TH) are main regulators of BAT development and function. However, it remains unknown how the magnetic resonance (MR)-based proton density fat fraction (PDFF) of supraclavicular adipose tissue used as a surrogate marker for BAT presence relates to $\mathrm{TH}$. Therefore, the purpose of this analysis was to investigate the relationship between supraclavicular PDFF and serum levels of TH. Methods: In total, 96 adult volunteers from a large cross-sectional study who underwent additional MR examination of the neck and pelvis were included in this analysis. Segmented PDFF maps of the supraclavicular and gluteal subcutaneous adipose tissue were generated. Delta PDFF was calculated as the difference between gluteal and supraclavicular PDFF and grouped as high $(\geq 12 \%)$ or low $(<12 \%)$ based on the median and the clinical rationale of a high versus low probability of BAT being present. Thyroid-stimulating hormone $(\mathrm{mlU} / \mathrm{L})$, free triiodothyronine $(\mathrm{FT} 3, \mathrm{pg} / \mathrm{mL})$ and free thyroxine (FT4, ng/dL) levels were determined in blood samples. Body mass index (BMI) was calculated as weight $(\mathrm{kg}) /$ height $(\mathrm{m})^{2}$. Statistical analyses included the use of paired samples $t$ test, simple linear regression analysis and a multivariable linear regression analysis. Results: The median age of the subjects ( $77 \%$ female) was 33 years, BMI ranged from 17.2 to $43.1 \mathrm{~kg} / \mathrm{m}^{2}$. Supraclavicular and gluteal PDFF differed significantly $(76.5 \pm 4.8$ vs. $89.4 \pm 3.5 \%, p<0.01)$.
\end{abstract}


Supraclavicular PDFF was associated with FT3 in subjects with high delta PDFF $\left(R^{2}=0.17, p<\right.$ $0.01)$, with higher FT3 being associated with lower supraclavicular PDFF $(y=85.2+-3.6 x)$. In a multivariable linear regression analysis considering further potential prognostic factors, the interaction between the delta PDFF group and FT3 remained a predictor for supraclavicular PDFF ( $B=-4.65, p<0.01)$. Discussion/Conclusions: Supraclavicular PDFF corresponds to the presence of BAT. In the present analysis, supraclavicular PDFF is correlated with FT3 in subjects with high delta PDFF. Therefore, the present findings suggest that biologically active T3 may be involved in the development of supraclavicular BAT.

(C) 2020 The Author(s)

Published by S. Karger AG, Basel

\section{Introduction}

Brown adipose tissue (BAT) is an organ contributing to thermoregulation in mammals including humans. This extensively vascularized tissue is characterized by smaller adipocytes than those in white adipose tissue (WAT), a high number of mitochondria, a centrally located nucleus, multiple small triglyceride droplets and more intracellular water. Brown adipocytes express uncoupling protein 1 , a protein uncoupling oxidative phosphorylation from adenosine triphosphate production, resulting in release of energy as heat. Depots of BAT in adult humans can be mostly found in the supraclavicular and cervical region [1]. Notably, there is evidence that adults exhibit two types of BAT having a rather similar cell morphology but originate from different progenitors: "classic" BAT and "beige" or "brown-in-white/brite" fat. Several studies suggest that within WAT depots, "browning," i.e. the process in which precursor cells placed in WAT become beige/brite cells instead of white adipocytes, can be induced [2-4]. Activation of BAT and browning of WAT contribute to an increase in total energy expenditure $[5,6]$, and several studies have investigated BAT as a potential target tissue for body fat reduction, reduction of elevated blood triglyceride concentrations and diabetes [7-9].

Besides approaches like cold exposure to increase BAT activity and volume or even to induce browning of WAT $[10,11]$, thyroid hormones (TH) have been shown to play a role in BAT function and expansion [12-14]. In humans, hyperthyroidism correlates with increased BAT activity and browning of WAT [15-19]. A cell-autonomous role of TH in regulating BAT function has been postulated [18, 20-22]. In particular, triiodothyronine (T3) has been described to not only activate BAT but also to regulate BAT differentiation and induce browning of WAT $[12,23,24]$.

So far, human studies on correlations between TH and BAT used [18F]-2-fluoro-2-deoxyglucose positron emission tomography (FDG-PET) and computed tomography (CT) for BAT detection based on its glucose uptake [15-17]. Recent developments established magnetic resonance imaging (MRI) as noninvasive imaging modality for detecting and characterizing BAT. In contrast to FDG-PET, MRI is independent from the current metabolic activity of BAT, free of ionizing radiation and without the need for intravenous contrast. Several studies have demonstrated MRI to offer powerful tools in BAT imaging [25-31]. The most frequently used approaches are chemical shift encoding-based fat quantification techniques [32, 33]. The state-of-the-art chemical shift encoding-based fat quantification technique considering multiple confounding factors relies on the measurement of the proton density fat fraction (PDFF), defined as the proportion of mobile proton density in fat tissue attributable to fat [34], as BAT has been shown to have a lower fat fraction compared to WAT [25, 35-37]. Therefore, PDFF can be used as surrogate marker for the presence of BAT. Furthermore, it can be assumed that a smaller PDFF difference between (potentially BATcontaining) supraclavicular and (white) subcutaneous adipose tissue means a higher resemblance of the adipose tissue composition within the two compartments. In other

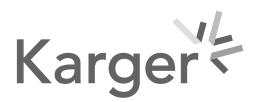


words, the more the PDFF values in supraclavicular and subcutaneous adipose tissue converge, the more the composition of supraclavicular adipose tissue, which is typically a mixture of BAT and WAT, resembles that of subcutaneous WAT. Reversely, a high PDFF difference (delta PDFF) between the two compartments reflects a different adipose tissue composition in supraclavicular compared to subcutaneous fat, presumably due to a larger proportion of BAT in the supraclavicular fat depot [25, 38, 39]. However, PDFF of WAT is associated with anthropometric parameters such as body mass index (BMI) and can therefore vary interindividually [40]. Thus, WAT PDFF can be used as intraindividual reference value for PDFF of WAT. Based on this reference, leading to an individual "delta PDFF," the supraclavicular adipose tissue composition can be interpreted as comparable to that of WAT or different from WAT.

To date, it remains unknown how the MRI-based PDFF of adipose tissue relates to TH levels. Thus, the purpose of the present analysis was to investigate the relationship between the PDFF of supraclavicular adipose tissue and TH levels. The supraclavicular adipose tissue was selected as a typical location where BAT might be present in humans, and the gluteal region represents a typical location enclosing primarily white adipocytes.

\section{Subjects and Methods}

\section{Subjects}

For a subgroup of subjects ( $n=111 ; 75$ women and 36 men) who participated in a crosssectional study at the Institute for Nutritional Medicine, Klinikum rechts der Isar, Technical University of Munich [41], additional MRI data are available. The aim of the cross-sectional study was to investigate genetic factors and resting metabolic rate. Study protocols and procedures were approved by the ethical committee of the School of Medicine of the Technical University of Munich, Germany. Subjects were screened for eligibility and were included if age was equal to or greater than 18 years. Participants who appeared healthy according to self-reporting, showed no history of or acute severe diseases (e.g., cancer, renal dialysis, inflammatory bowel disease, stroke) or surgery within the last 3 months and did not have acute physical impairment were eligible for the study. The study design did not define explicit inclusion criteria. Based on collected data on health status and lifestyle factors, this provided the possibility to include and exclude participants for specific research questions. Pregnant women as well as subjects with standard contraindications for MRI examinations were excluded from the additional MRI scan. For the current analysis, subjects who did not complete MRI $(n=4)$, in whom blood sampling was not possible $(n=5)$, with prior complete thyroidectomy ( $n=1)$, with Graves' disease $(n=1)$ and with levels of thyroid-stimulating hormone (TSH) of $<0.1 \mathrm{mIU} / \mathrm{L}$, i.e. below detection limit, indicating hyperthyroidism $(n=3)$ were excluded. Furthermore, one subject with a clearly outlying free thyroxine (FT4) level was excluded. In total, 96 participants were included in the present statistical analysis. All subjects gave written informed consent.

\section{BMI Measurement}

Height was measured without shoes in a standing position using a stadiometer (Seca, Hamburg, Germany) and reported to the nearest $0.1 \mathrm{~cm}$. Weight was assessed during a bioimpedance analysis using Tanita BC 418 MA (Tanita, Tokyo, Japan). Measurements were performed in light clothing and with voided bladder, whereby $1.0 \mathrm{~kg}$ was subtracted automatically for clothes. BMI was calculated as the quotient of weight in kilogram and height in meters squared $\left(\mathrm{kg} / \mathrm{m}^{2}\right)$. Information on the health status and medication was assessed using standardized questionnaires.

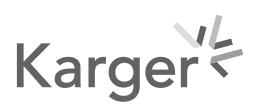


Junker et al.: PDFF in Supraclavicular Fat and Thyroid Hormones

TH Analysis

Fasting serum samples were analyzed by SYNLAB MVZ Labor München Zentrum GbR (Munich, Germany) for FT4 (ng/dL) and free triiodothyronine (FT3, pg/mL) using chemiluminescence immunoassay (CLIA, Siemens Centaur XP; Siemens Healthcare GmbH, Erlangen, Germany) according to standard operation procedures. The analytical sensitivity of the test was $0.1 \mathrm{ng} / \mathrm{dL}$ for FT4 and $0.2 \mathrm{pg} / \mathrm{mL}$ for FT3. The tests were standardized according to internal standards using United States Pharmacopeia. The coefficients of variations (C.V.) between and within runs were as follows for FT4 and FT3: FT4, 2.23-3.33\% (within runs) and 3.44-4.58\% (between runs); FT3, 2.35-3.08\% (within runs) and 2.76-4.05\% (between runs). The reference values specified by the laboratory are as follows: $0.89-1.76 \mathrm{ng} / \mathrm{dL}$ for FT4 and 2.3-4.2 pg/mL for FT3. The TSH levels (mlU/L) were determined in duplicate using an antibody enzyme-linked immunosorbent assay (HUMAN Diagnostic, USA). According to the manufacturer's protocol, the reference values are determined within 0.3-4.0 mlU/L TSH. The analytical sensitivity of the test was determined for $<0.10 \mathrm{mlU} / \mathrm{L} \mathrm{TSH}$. The standard curve was calibrated with the 2nd IRP WHO Reference Standard 80/558. Furthermore, the standard curve was based on TSH concentrations across a range of $0-30 \mathrm{mlU} / \mathrm{L}$. The C.V. between (C.V. $=2.5 \%)$ and within runs (C.V. $=4.7 \%)$ were below their advised levels. A monoclonal TSH antibody was pre-coated on a microtiter plate. Serum samples, calibrators and enzyme conjugates were added forming a sandwich complex fixed by binding to the immobilized antibody. After washing out excess components, a substrate reagent was added, and the resulting color was measured photometrically (Infinite F50; Tecan GmbH, Crailsheim, Germany) at a wavelength of $450 \mathrm{~nm}$. The optical imprecisions were corrected by the reference wavelength of $620 \mathrm{~nm}$. Interpolation was used (HUMAN Diagnostic, USA) for the calculation of TSH concentrations using Graphpad Prism 7 (GraphPad Software, USA).

\section{MRI Measurements}

Subjects underwent an MRI examination of the neck and the abdomen/pelvis on a 3T Philips scanner (Ingenia; Philips Healthcare, Best, Netherlands) using a head-neck coil and a combination of anterior and posterior coil arrays, respectively. Scans were performed in an air-conditioning-controlled scanner room $\left(21^{\circ} \mathrm{C}\right)$ after some acclimatization time (altogether approximately $45 \mathrm{~min}$ ), while the study procedure was explained and the scanning preparations were conducted. The scans were performed throughout all seasons.

Supraclavicular and Pelvic PDFF Mapping

In order to determine the supraclavicular and gluteal subcutaneous PDFF, a scan protocol as described in detail before [40] was used, including two six-echo multi-echo 3D spoiled gradient echo sequences with bipolar gradients of the supraclavicular and the posterior upper pelvic region. Sequence parameters are listed in online supplementary Table S1 (for all online suppl. material, see www.karger.com/doi/10.1159/000507294). A small flip angle was used to minimize T1 bias effects [42, 43]. PDFF maps were generated online using the fat quantification routine of the MR vendor [44]. This routine comprises a complex-based waterfat separation algorithm, accounting for known confounding factors including phase error correction, a single $\mathrm{T} 2 *$ correction and the consideration of the spectral complexity of fat using the multi-peak fat spectrum model of Ren et al. [45]. The imaging-based PDFF map was computed as the ratio of the fat signal over the sum of fat and water signals.

PDFF Analysis

By the use of an in-house semiautomatic segmentation tool implemented in MATLAB (MathWorks, Natick, MA, USA), a radiologist (6 years of experience in whole-body imaging) segmented the complete supraclavicular and the complete deep gluteal fat depots bilaterally,

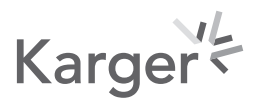


as described before in detail [40]. PDFF values of the resulting volumes of interest (in \%) were noted separately for the left and right fat depot, and the average PDFF per region (supraclavicular/gluteal) was calculated. Reproducibility of the segmentation was shown in a previous publication for the same adipose tissue compartments [36]. Delta PDFF was calculated by subtracting the average supraclavicular PDFF from the average gluteal subcutaneous PDFF. Two approximately equally sized groups were defined: a high delta PDFF group with delta PDFF $\geq 12 \%(n=51)$ and a low delta PDFF group with PDFF $<12 \%(n=45)$. The cut-off of $12 \%$ was the median delta PDFF rounded to the nearest integer. The groups were built in order to differentiate between subjects where the supraclavicular depot showed a distinctly different composition compared to WAT, pointing to a high probability of BAT being present, and subjects where the supraclavicular adipose tissue composition resembled that of WAT, i.e. with a low probability of BAT being present. The clinical rationale for choosing the rounded median delta PDFF were findings from previous studies showing the difference between mean fat fraction values in BAT versus WAT to lie between $8 \%$ in middle-aged adults and $22 \%$ in children $[25,38]$. Thus, $12 \%$ was selected as a reasonable cut-off for the present sample of adults with a median age of 33 years.

\section{Statistical Analysis}

The collected parameters were normally distributed with the exception of age, weight, BMI and TSH; thus, data are expressed as mean \pm standard deviation (range in parentheses), if not otherwise denoted. Paired samples $t$ test was used for comparison of PDFF values of the supraclavicular and subcutaneous gluteal adipose tissue compartment. Associations between supraclavicular PDFF and TH (FT3, FT4 and TSH) levels were evaluated using simple linear regression analysis and Spearman's rank correlation, respectively (for normal and non-normal distributed parameters). Each analysis was first performed for all subjects and then separately for the low delta PDFF group and the high delta PDFF group (low: delta PDFF <2\%; high: delta PDFF $\geq 12 \%$ ). Subsequently, a multivariable linear regression analysis was performed. The analysis was carried out with supraclavicular PDFF as the dependent variable, and age, sex, BMI, FT3, FT4, TSH, delta PDFF group and the interaction term between delta PDFF group and FT3 as independent variables. This interaction term was supposed to reflect a possible difference in the effect of FT3 on supraclavicular PDFF depending on whether the supraclavicular adipose tissue depot resembles WAT or possesses a different composition, pointing to BAT. Statistical analysis was performed by using MedCalc Statistical Software (version 16.4.3; MedCalc Software bvba, Ostend, Belgium; https://www.medcalc.org; 2016) and the software package SPSS (IBM SPSS Statistics for Windows, Version 25.0; IBM Corporation, Armonk, NY, USA). All tests were performed with a significance level of 0.05 (two-sided), and no correction was made for multiple testing due to the explorative character of the analysis.

\section{Results}

In total, 96 volunteers were included in the analysis ( 64 women and 32 men). The subjects had a broad range of BMI (17.2-43.1 kg/ $\mathrm{m}^{2}$ ). Median age was 33 years (range, 21.2-77.3) (Table 1). Median age in women was 30.9 years (range, 21.2-77.3) and in men 34.4 years (range, 22.6-61.3). Within the high and the low delta PDFF groups, sex, age and BMI were distributed as follows: in the low delta PDFF group, there were 26 women (58\%), median age was 44.6 years and mean BMI was $28.2 \mathrm{~kg} / \mathrm{m}^{2}$. In the high delta PDFF group, there were 38 women (75\%), median age was 27.9 years and mean BMI was $23.0 \mathrm{~kg} / \mathrm{m}^{2}$. Of the 64 women included in the analysis, 26 were on oral contraceptives. None of the subjects was on statins or took biotin as supplement.

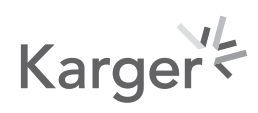


Table 1. Characteristics of the study population $(n=96)$

Females, $n(\%)$

Age, years

Weight, $\mathrm{kg}$

Height, $\mathrm{cm}$

$\mathrm{BMI}, \mathrm{kg} / \mathrm{m}^{2}$

FT3, $\mathrm{pg} / \mathrm{mL}$

FT4, ng/dL

$\mathrm{TSH}, \mathrm{mlU} / \mathrm{L}$

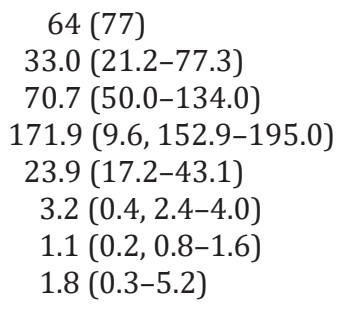

Data are presented as median (range), mean (SD, range), or as stated. BMI, body mass index; FT3, free triiodothyronine; FT4, free thyroxine; TSH, thyroid stimulating hormone; SD, standard deviation.

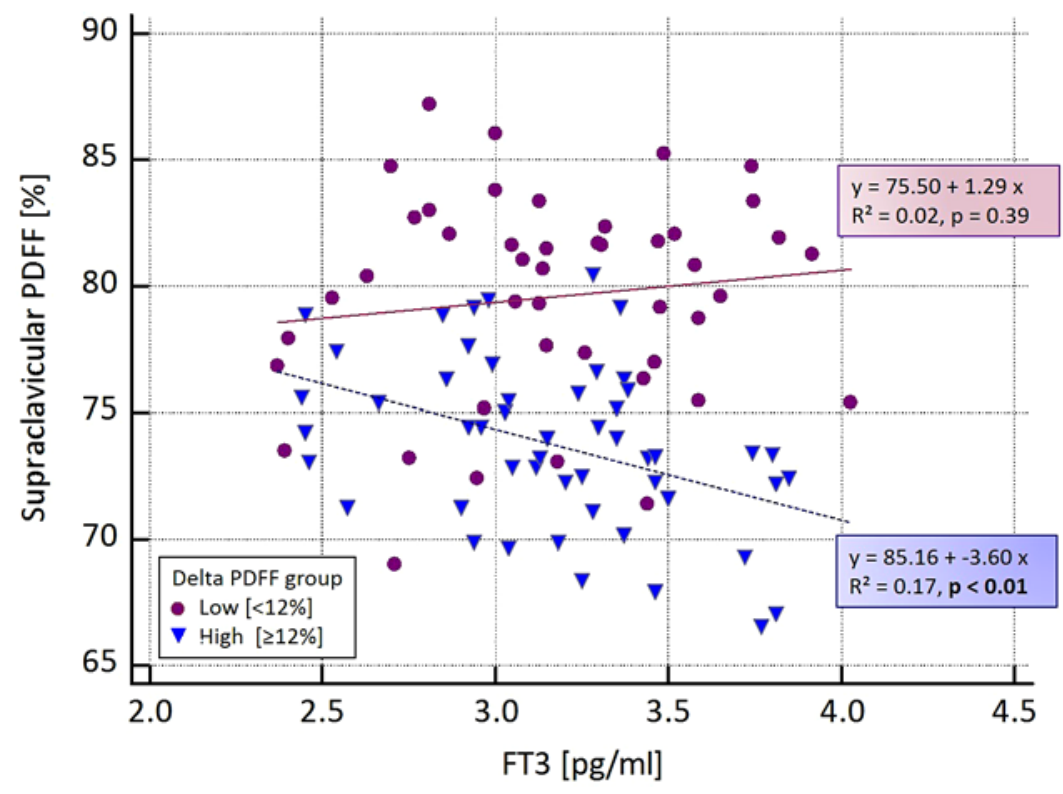

Fig. 1. Scatter plot depicting the association between free triiodothyronine (FT3) and supraclavicular proton density fat fraction (PDFF) for the high delta PDFF (blue triangles) versus low delta PDFF (violet dots) group. The high delta PDFF group shows a significant association $\left(R^{2}=0.17, p<0.01\right)$, while the association in the low delta PDFF group is not significant $\left(R^{2}=0.02, p=0.39\right)$.

Supraclavicular PDFF with $76.5 \pm 4.8 \%(66.5-87.2 \%)$ was significantly lower $(p<0.01)$ than subcutaneous gluteal PDFF with $89.4 \pm 3.5 \%$ (78.7-94.8\%). Mean delta PDFF was 12.9 $\pm 4.1 \%(3.2-22.7 \%)$. The unilateral volume of the segmented deep gluteal fat depot ranged from 135.6 to $780 \mathrm{~mL}$, the unilateral volume of the segmented supraclavicular fat depot ranged from 15.9 to $156.7 \mathrm{~mL}$. Correlations between PDFF values and age, sex and BMI are shown in online supplementary Table S2.

Simple linear regression analyses did not show significant associations between the supraclavicular PDFF and FT3 when testing for the entirety of the subjects $\left(R^{2}=0.007, p=\right.$ 0.41 ). When looking separately at the two delta PDFF groups, regression analysis revealed a significant association between supraclavicular PDFF and FT3 in the subjects with high delta PDFF $\left(R^{2}=0.17, p<0.01\right)$, whereas no association was observed for the low delta PDFF subgroup $\left(R^{2}=0.02, p=0.39\right.$ ) (Fig. 1$)$. Figure 2 shows examples of PDFF maps of 2 subjects 


\begin{tabular}{ll|l}
\cline { 2 - 2 } Obes Facts 2020;13:331-343 \\
\cline { 2 - 3 } & DOI: 10.1159/000507294 & $\begin{array}{l}\text { @ 2020 The Author(s). Published by S. Karger AG, Basel } \\
\text { www.karger.com/ofa }\end{array}$ \\
\cline { 2 - 3 }
\end{tabular}

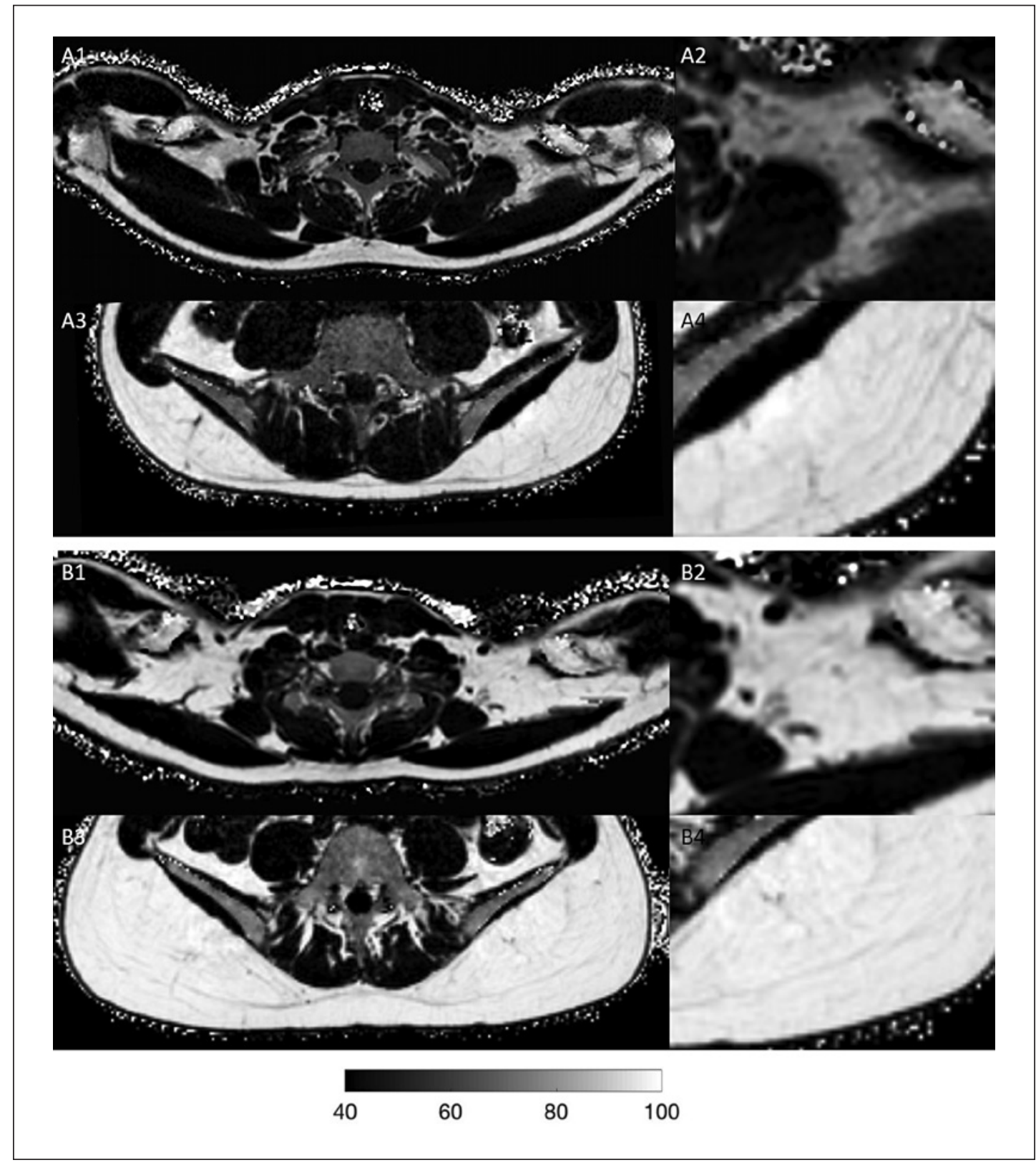

Fig. 2. A Supraclavicular/zoomed supraclavicular (A1/A2) and gluteal/zoomed gluteal proton density fat fraction (PDFF) map (A3/A4) in a 23-year-old male subject (body mass index [BMI]: $19.3 \mathrm{~kg} / \mathrm{m}^{2}$ ). Supraclavicular PDFF was $67 \%$, gluteal PDFF was $89 \%$, resulting in a delta PDFF of $22 \%$. Free triiodothyronine (FT3) was high with $3.81 \mathrm{pg} / \mathrm{mL}$. B Supraclavicular/zoomed supraclavicular (B1/B2) and gluteal/zoomed gluteal PDFF map (B3/ B4) in a 24-year-old female subject (BMI: $20.6 \mathrm{~kg} / \mathrm{m}^{2}$ ). Supraclavicular PDFF was $78 \%$, gluteal PDFF was $92 \%$, resulting in a delta PDFF of $14 \%$. FT3 was low with $2.45 \mathrm{pg} / \mathrm{mL}$. Note that both subjects have a high delta PDFF. Subject A with a high FT3 has a lower supraclavicular PDFF compared to subject B with a low FT3. Greyscale bar shows PDFF values within the tissues.

from the high delta PDFF group with high and low FT3: the subject with high FT3 has a lower supraclavicular PDFF compared to the subject with low FT3. There were no significant associations between supraclavicular PDFF and FT4 in the entire population $\left(R^{2}=0.002, p=0.69\right)$, the high delta group $\left(R^{2}=0.04, p=0.18\right)$ or the low delta group $\left(R^{2}=0.0009, p=0.85\right)$. The 
Table 2. Results of the multivariable linear regression analysis with supraclavicular PDFF as dependent variable

\begin{tabular}{lccr}
\hline Independent variables & Coefficient B & Significance $p$ & $r_{\text {partial }}$ \\
\hline FT3 & 1.98 & ns $(0.08)$ & 0.19 \\
FT4 & -1.28 & ns $(0.52)$ & -0.07 \\
TSH & -0.14 & ns $(0.66)$ & -0.05 \\
Age & 0.06 & $\mathbf{0 . 0 1}$ & 0.26 \\
BMI & 0.37 & $<\mathbf{0 . 0 1}$ & 0.56 \\
Sex & 0.38 & ns $(0.60)$ & 0.06 \\
Interaction term (delta PDFF group $\times$ FT3) & -4.65 & $<\mathbf{0 . 0 1}$ & -0.31 \\
Delta PDFF group & 11.54 & $\mathbf{0 . 0 2}$ & 0.25 \\
\hline
\end{tabular}

Adjusted $R^{2}=0.64$. PDFF, proton density fat fraction; FT3, free triiodothyronine; FT4, free thyroxine; TSH, thyroid stimulating hormone; BMI, body mass index; ns, nonsignificant.

same was true for TSH and PDFF values: no association was found for TSH and the supraclavicular PDFF in the entire group or the in high or the low delta group (rho $=-0.13, p=0.21$; rho $=0.03, p=0.85$ and rho $=-0.03, \mathrm{p}=0.84$, respectively). Online supplementary Figure $\mathrm{S} 1$ shows the plots of the associations between supraclavicular PDFF and FT4 and TSH, respectively. Correlation analyses of gluteal PDFF and delta PDFF with TH did not result in any significant associations (online suppl. Table S3).

The results of the multivariable linear regression analysis considering age, sex, BMI, FT3, FT4, TSH, delta PDFF group and the interaction term between delta PDFF group and FT3 revealed that BMI and the interaction between FT3 and the delta PDFF group were the strongest drivers for supraclavicular PDFF, next to the weaker driver age. Detailed results are presented in Table 2 .

\section{Discussion}

The present analysis provides evidence for a significant difference between the PDFF in the supraclavicular and subcutaneous fat depots, which is likely due to a difference in the water-fat cellular composition between the two locations. Furthermore, PDFF in the potentially BAT-containing supraclavicular adipose tissue was inversely correlated with FT3 levels in subjects with a high compositional difference between supraclavicular and subcutaneous PDFF. In a multivariable linear regression analysis, the interaction between FT3 and the PDFF difference (delta PDFF) was one of the main drivers of supraclavicular PDFF.

Regarding the methodology of using adipose tissue PDFF as surrogate marker for the presence of BAT, the significantly lower PDFF in supraclavicular adipose tissue compared to subcutaneous gluteal adipose tissue is in line with the findings of other studies, suggesting different tissue compositions in supraclavicular compared to subcutaneous adipose tissue $[25,32,38,40,46,47]$. The lower PDFF in the supraclavicular region indicates a reduced lipid content. A highly likely explanation for this is the presence of BAT $[38,40]$, which is known to have lower lipid content and more mitochondria compared to WAT. Moreover, the supraclavicular fat compartment is the most common region in adults for BAT detected in PET/CT scans $[48,49]$. With this knowledge, the use of PDFF as surrogate marker for the presence of BAT seems reasonable. However, other explanations for differences in PDFF values need to be taken into account, such as variations in vascularization. It has been shown previously that PDFF in WAT is associated with BMI and can thus vary from individual to individual [40].

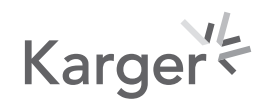


Based on this knowledge, subcutaneous WAT was used as an intraindividual reference value in the present analysis in order to further classify the supraclavicular PDFF with regard to the presence of BAT. This intraindividual reference is expressed as delta PDFF, i.e. the difference between supraclavicular and gluteal subcutaneous (i.e., WAT) PDFF. A high delta PDFF reflects a markedly different composition of supraclavicular adipose tissue compared to subcutaneous WAT, while similar PDFF values in supraclavicular and gluteal PDFF point to the supraclavicular fat depot resembling a WAT depot.

A central finding of the present analysis is the inverse correlation between supraclavicular PDFF as surrogate marker for BAT presence and serum levels of FT3 in subjects with a high delta PDFF, i.e. a large compositional difference between the supraclavicular and the subcutaneous adipose tissue. When performing a multivariable linear regression analysis for further verification of the aforementioned finding under consideration of other potentially relevant parameters, an interaction term was created to expand the understanding of the relationship between FT3, delta PDFF and supraclavicular PDFF and to express that the effect between FT3 and supraclavicular PDFF is different for the two different delta PDFF categories, i.e. for subjects with a rather different or rather similar adipose tissue in the supraclavicular region compared to WAT. Thereby, it was confirmed that the interaction between high FT3 and high delta PDFF is associated with lower supraclavicular PDFF, thus again presumably with more BAT. This assumed influence of TH on BAT is consistent with results from various PET/CT studies, where higher serum TH levels (as in hyperthyroidism) were associated with more metabolically active BAT or higher glucose uptake of BAT in rodents $[18,20,21]$ and humans $[15,16,50-52]$. While some studies could not confirm those findings $[17,53,54]$, this dissent might be caused by the methodology of PET/CT, with BAT activity and visibility on PET images being dependent on a number of factors such as room temperature. T3 values have been shown to affect BAT by activating thermogenesis and increasing BAT volume $[12-14,55]$. However, in the present analysis, the influence of FT3 on supraclavicular adipose tissue PDFF becomes only visible when focusing on subjects with a high delta PDFF, i.e. with a high compositional difference between supraclavicular adipose tissue and WAT, presumably due to higher proportions of BAT being present. In cases of a high resemblance of the supraclavicular adipose tissue with pure WAT depots, i.e. non-existent or a very low amount of BAT, no effect of FT3 is visible. This is the case in the subjects with a low delta PDFF, where we did not find an association between FT3 and supraclavicular PDFF.

The lack of a correlation between supraclavicular PDFF and FT4 as compared to FT3 is likely due to $\mathrm{T} 4$ being a prohormone and $\mathrm{T} 3$ being the active metabolite regulating the differentiation of adipocytes as well as being the factor necessary for BAT function [24, 56, 57]. A correlation with TSH might also be expected, but as our cohort consists of individuals in the broad euthyroid range of TSH, the visibility of such a correlation probably depends on the sample size, which is in this context relatively small in the current study.

The finding that beside the interaction term, BMI and age are drivers of supraclavicular PDFF with lower BMI and younger age leading to lower supraclavicular PDFF, thus presumably more BAT, is consistent with results from PET/CT studies showing lower BMI and younger age being associated with more (activated) BAT [49, 58-60].

The development of brown/beige adipocytes and expansion of BAT is under the complex and dynamic control of a variety of factors with activation of the $\beta_{3}$-adrenoceptors by catecholamines being the most important ones [61]. The potential role of TH in this context is still poorly understood. There is some evidence that T3 has a direct stimulatory effect on BAT thermogenesis $[21,24,62]$, but there is also limited evidence that $\mathrm{T} 3$ may exert a central action resulting in an activation of the sympathetic nerve system [12]. Other data indicate that at least under experimental hyperthyroidism TH promote the browning of WAT [19]. The results of our analysis suggest a link between biologically active $\mathrm{T} 3$ and the presence of supraclavicular BAT, but the 
Junker et al.: PDFF in Supraclavicular Fat and Thyroid Hormones

effect only becomes visible in subjects with presumed higher proportions of supraclavicular BAT under physiological conditions. However, in a state of hyperthyroidism, elevated biologically active T3 may have a much stronger impact on BAT development and function.

\section{Strengths and Limitations}

One strength of the present analysis is the relatively high number of volunteers included, compared to other studies that are mostly based on PET imaging with smaller patient numbers or with a retrospective design $[15,17,53,59]$. Secondly, imaging of BAT based on PET data relies on the current metabolic activity of the tissue, while MRI provides a more robust parameter - the PDFF - for differentiating BAT from WAT, independent from the metabolic status.

The present analysis also has some limitations. First, histology was not used as a gold standard for characterizing adipose tissue. Without adipose tissue biopsy samples, it is not possible to ultimately substantiate the presence of BAT as well as the cellular characteristics of the depots under investigation. Second, partial volume effects need to be taken into account when performing PDFF measurements with MRI, as due to voxel size limitations, PDFF cannot differentiate between intracellular water content and non-lipid tissue portions (e.g., from vessels) within a voxel. The isotropic voxel size in the data set was $1.5 \mathrm{~mm}$ in each dimension. Thus, partial volume effects from very small vessels, lymph nodes and adjacent muscles cannot be excluded, and those effects are possibly larger in the supraclavicular adipose tissue compared to the subcutaneous adipose tissue due to the distinct anatomy. Furthermore, PDFF analyses only present average values of volumes of interest and thus cannot discriminate between a cluster of brown adipocytes and mixed clusters of white and brown adipocytes. Third, 26 of the 64 women included in the analysis were on oral contraceptives, which should be taken into account, as a possible influence of oral contraceptives on thyroid function is still being discussed $[63,64]$. Lastly, deiodinase activity in BAT was not measured. As deiodinase is considered an enzyme relevant in BAT physiology [62, 65], not evaluating deiodinase activity in the current analysis can be considered a potential limitation.

\section{Conclusion}

In conclusion, this analysis shows a significant difference between supraclavicular and subcutaneous adipose tissue composition as indicated by MRI-based PDFF, suggesting the presence of BAT in the supraclavicular fat compartment. Regarding TH, PDFF in the potentially BAT containing supraclavicular adipose tissue was inversely correlated with FT3 in subjects with a high difference between supraclavicular and subcutaneous PDFF (a high delta PDFF), i.e. a large compositional difference in the supraclavicular adipose tissue compared to the individual's WAT. The interaction between FT3 and delta PDFF remained a driver for supraclavicular PDFF in a multivariable linear regression analysis that also took into account age, sex, BMI, FT3, FT4, TSH and delta PDFF group. Therefore, the present findings suggest that levels of FT3 might possibly influence the presence of supraclavicular BAT; however, this effect only becomes visible in subjects with larger compositional differences between WAT and the supraclavicular adipose tissue, i.e. with presumably higher proportions of BAT in supraclavicular adipose tissue.

\section{Acknowledgements}

The authors are grateful to all persons participating in the study as well as to the students involved in the study.

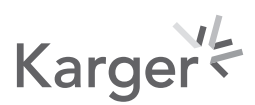


Junker et al.: PDFF in Supraclavicular Fat and Thyroid Hormones

\section{Statement of Ethics}

All procedures performed were in accordance with the ethical standards of the institutional research committee and with the 1964 Helsinki Declaration and its later amendments or comparable ethical standards. Written informed consent was obtained from all subjects prior to data collection. Study protocols and procedures were approved by the ethical committee of the Faculty of Medicine of the Technical University of Munich, Germany.

\section{Disclosure Statement}

Dimitrios Karampinos receives grant support from Philips Healthcare. The remaining authors declare no conflicts of interest.

\section{Funding Sources}

The present work was supported by the European Research Council (grant agreement No. 677661, ProFatMRI), Philips Healthcare, the Else Kroener-Fresenius Foundation, Bad Homburg, Germany, and the Helmholtz cross-program topic "Metabolic Dysfunction." This work reflects only the authors' view and the EU is not responsible for any use that may be made of the information it contains.

\section{Author Contributions}

Daniela Junker: concept of study design, data acquisition, data segmentation, statistical analysis, drafting of manuscript. Jan Syväri: data acquisition, critical revision of manuscript. Dominik Weidlich: data acquisition, data postprocessing, critical revision of manuscript. Christina Holzapfel: data acquisition, critical revision of manuscript. Theresa Drabsch: data acquisition, critical revision of manuscript. Birgit Waschulzik: statistical analysis, critical revision of manuscript. Ernst J. Rummeny: concept of study design, critical revision of manuscript. Hans Hauner: concept of study design, critical revision of manuscript. Dimitrios $C$. Karampinos: concept of study design, data acquisition, data postprocessing, statistical analysis, critical revision of manuscript.

\section{References}

1 Enerbäck S. Human brown adipose tissue. Cell Metab. 2010 Apr;11(4):248-52.

2 Harms M, Seale P. Brown and beige fat: development, function and therapeutic potential. Nat Med. 2013 Oct; 19(10):1252-63.

3 Bartelt A, Heeren J. Adipose tissue browning and metabolic health. Nat Rev Endocrinol. 2014 Jan;10(1):24-36

4 Fisher FM, Kleiner S, Douris N, Fox EC, Mepani RJ, Verdeguer F, et al. FGF21 regulates PGC-1 $\alpha$ and browning of white adipose tissues in adaptive thermogenesis. Genes Dev. 2012 Feb;26(3):271-81.

5 Ouellet V, Labbé SM, Blondin DP, Phoenix S, Guérin B, Haman F, et al. Brown adipose tissue oxidative metabolism contributes to energy expenditure during acute cold exposure in humans. J Clin Invest. 2012 Feb;122(2):545-52.

6 Shabalina IG, Petrovic N, de Jong JM, Kalinovich AV, Cannon B, Nedergaard J. UCP1 in brite/beige adipose tissue mitochondria is functionally thermogenic. Cell Rep. 2013 Dec;5(5):1196-203.

7 Gunawardana SC, Piston DW. Insulin-independent reversal of type 1 diabetes in nonobese diabetic mice with brown adipose tissue transplant. Am J Physiol Endocrinol Metab. 2015 Jun;308(12):E1043-55.

8 Bartelt A, Bruns OT, Reimer R, Hohenberg H, Ittrich H, Peldschus K, et al. Brown adipose tissue activity controls triglyceride clearance. Nat Med. 2011 Feb;17(2):200-5.

9 Yoneshiro T, Saito M. Activation and recruitment of brown adipose tissue as anti-obesity regimens in humans. Ann Med. 2015 Mar;47(2):133-41. 


\begin{tabular}{|c|c|}
\hline Obes Facts 2020;13:331-343 & \\
\hline DOI: 10.1159/000507294 & $\begin{array}{l}\text { (c) } 2020 \text { The Author(s). Published by S. Karger AG, Basel } \\
\text { www.karger.com/ofa }\end{array}$ \\
\hline
\end{tabular}

Junker et al.: PDFF in Supraclavicular Fat and Thyroid Hormones

10 Fedorenko A, Lishko PV, Kirichok Y. Mechanism of fatty-acid-dependent UCP1 uncoupling in brown fat mitochondria. Cell. 2012 Oct;151(2):400-13.

11 Klingenspor M. Cold-induced recruitment of brown adipose tissue thermogenesis. Exp Physiol. 2003 Jan; 88(1):141-8.

12 Cioffi F, Gentile A, Silvestri E, Goglia F, Lombardi A. Effect of Iodothyronines on Thermogenesis: Focus on Brown Adipose Tissue. Front Endocrinol (Lausanne). 2018 May; 9:254.

13 Santhanam P, Ahima RS, Mammen JS, Giovanella L, Treglia G. Brown Adipose Tissue (BAT) detection by 18F-FDG PET and thyroid hormone level(s)-a systematic review. Endocrine. 2018 Nov;62(2):496-500.

14 Yau WW, Singh BK, Lesmana R, Zhou J, Sinha RA, Wong KA, et al. Thyroid hormone (T3) stimulates brown adipose tissue activation via mitochondrial biogenesis and MTOR-mediated mitophagy. Autophagy. 2019 Jan;15(1):131-50.

15 Lahesmaa M, Orava J, Schalin-Jäntti C, Soinio M, Hannukainen JC, Noponen T, et al. Hyperthyroidism increases brown fat metabolism in humans. J Clin Endocrinol Metab. 2014 Jan;99(1):E28-35.

16 Broeders EP, Vijgen GH, Havekes B, Bouvy ND, Mottaghy FM, Kars M, et al. Thyroid Hormone Activates Brown Adipose Tissue and Increases Non-Shivering Thermogenesis-A Cohort Study in a Group of Thyroid Carcinoma Patients. PLoS One. 2016 Jan;11(1):e0145049.

17 Gavrila A, Hasselgren PO, Glasgow A, Doyle AN, Lee AJ, Fox P, et al. Variable Cold-Induced Brown Adipose Tissue Response to Thyroid Hormone Status. Thyroid. 2017 Jan;27(1):1-10.

18 Weiner J, Kranz M, Klöting N, Kunath A, Steinhoff K, Rijntjes E, et al. Thyroid hormone status defines brown adipose tissue activity and browning of white adipose tissues in mice. Sci Rep. 2016 Dec;6(1):38124.

19 Martínez-Sánchez N, Moreno-Navarrete JM, Contreras C, Rial-Pensado E, Fernø J, Nogueiras R, et al. Thyroid hormones induce browning of white fat. J Endocrinol. 2017 Feb;232(2):351-62.

20 Martinez de Mena R, Scanlan TS, Obregon MJ. The T3 receptor beta1 isoform regulates UCP1 and D2 deiodinase in rat brown adipocytes. Endocrinology. 2010 Oct;151(10):5074-83.

21 Bianco AC, Silva JE. Intracellular conversion of thyroxine to triiodothyronine is required for the optimal thermogenic function of brown adipose tissue. J Clin Invest. 1987 Jan;79(1):295-300.

22 Ribeiro MO, Bianco SD, Kaneshige M, Schultz JJ, Cheng SY, Bianco AC, et al. Expression of uncoupling protein 1 in mouse brown adipose tissue is thyroid hormone receptor-beta isoform specific and required for adaptive thermogenesis. Endocrinology. 2010 Jan;151(1):432-40.

23 Alvarez-Crespo M, Csikasz RI, Martínez-Sánchez N, Diéguez C, Cannon B, Nedergaard J, et al. Essential role of UCP1 modulating the central effects of thyroid hormones on energy balance. Mol Metab. 2016 Feb;5(4):271-82.

24 Bianco AC, McAninch EA. The role of thyroid hormone and brown adipose tissue in energy homoeostasis. Lancet Diabetes Endocrinol. 2013 Nov;1(3):250-8.

25 Franz D, Karampinos DC, Rummeny EJ, Souvatzoglou M, Beer AJ, Nekolla SG, et al. Discrimination Between Brown and White Adipose Tissue Using a 2-Point Dixon Water-Fat Separation Method in Simultaneous PET/ MRI. J Nucl Med. 2015 Nov;56(11):1742-7.

26 Borga M, Virtanen KA, Romu T, Leinhard OD, Persson A, Nuutila P, et al. Brown adipose tissue in humans: detection and functional analysis using PET (positron emission tomography), MRI (magnetic resonance imaging), and DECT (dual energy computed tomography). Methods Enzymol. 2014;537:141-59.

27 Romu T, Elander L, Leinhard OD, Lidell ME, Betz MJ, Persson A, et al. Characterization of brown adipose tissue by water-fat separated magnetic resonance imaging. J Magn Reson Imaging. 2015 Dec;42(6):1639-45.

$28 \mathrm{Hu}$ HH. Magnetic Resonance of Brown Adipose Tissue: A Review of Current Techniques. Crit Rev Biomed Eng. 2015;43(2-3):161-81.

29 Karampinos DC, Weidlich D, Wu M, Hu HH, Franz D. Techniques and Applications of Magnetic Resonance Imaging for Studying Brown Adipose Tissue Morphometry and Function. Handb Exp Pharmacol. 2019;251:299-324.

30 Sampath SC, Sampath SC, Bredella MA, Cypess AM, Torriani M. Imaging of Brown Adipose Tissue: state of the Art. Radiology. 2016 Jul;280(1):4-19.

31 Sun L, Yan J, Sun L, Velan SS, Leow MK. A synopsis of brown adipose tissue imaging modalities for clinical research. Diabetes Metab. 2017 Oct;43(5):401-10.

$32 \mathrm{Hu} \mathrm{HH}$, Yin L, Aggabao PC, Perkins TG, Chia JM, Gilsanz V. Comparison of brown and white adipose tissues in infants and children with chemical-shift-encoded water-fat MRI. J Magn Reson Imaging. 2013 0ct;38(4):885-96.

33 Rasmussen JM, Entringer S, Nguyen A, van Erp TG, Burns J, Guijarro A, et al. Brown adipose tissue quantification in human neonates using water-fat separated MRI. PLoS One. 2013 Oct;8(10):e77907.

34 Reeder SB, Hu HH, Sirlin CB. Proton density fat-fraction: a standardized MR-based biomarker of tissue fat concentration. J Magn Reson Imaging. 2012 Nov;36(5):1011-4.

35 Hu HH, Perkins TG, Chia JM, Gilsanz V. Characterization of human brown adipose tissue by chemical-shift water-fat MRI. AJR Am J Roentgenol. 2013 Jan;200(1):177-83.

36 Franz D, Diefenbach MN, Treibel F, Weidlich D, Syväri J, Ruschke S, et al. Differentiating supraclavicular from gluteal adipose tissue based on simultaneous PDFF and T2* mapping using a 20-echo gradient-echo acquisition. J Magn Reson Imaging. 2019 Aug;50(2):424-34.

37 McCallister A, Zhang L, Burant A, Katz L, Branca RT. A pilot study on the correlation between fat fraction values and glucose uptake values in supraclavicular fat by simultaneous PET/MRI. Magn Reson Med. 2017 Nov; 78(5):1922-32.

38 Franssens BT, Hoogduin H, Leiner T, van der GraafY, Visseren FL. Relation between brown adipose tissue and measures of obesity and metabolic dysfunction in patients with cardiovascular disease. J Magn Reson Imaging. 2017 Aug;46(2):497-504. 


\begin{tabular}{l|l}
\hline Obes Facts 2020;13:331-343 \\
\hline DOI: 10.1159/000507294 & $\begin{array}{l}\text { (c) 2020 The Author(s). Published by S. Karger AG, Basel } \\
\text { www.karger.com/ofa }\end{array}$ \\
\hline
\end{tabular}

Junker et al.: PDFF in Supraclavicular Fat and Thyroid Hormones

39 Hui SC, Ko JK, Zhang T, Shi L, Yeung DK, Wang D, et al. Quantification of brown and white adipose tissue based on Gaussian mixture model using water-fat and T2* MRI in adolescents. J Magn Reson Imaging. 2017 Sep; 46(3):758-68.

40 Franz D, Weidlich D, Freitag F, Holzapfel C, Drabsch T, Baum T, et al. Association of proton density fat fraction in adipose tissue with imaging-based and anthropometric obesity markers in adults. Int J Obes. 2018 Feb; 42(2):175-82.

41 Drabsch T, Holzapfel C, Stecher L, Petzold J, Skurk T, Hauner H. Associations Between C-Reactive Protein, Insulin Sensitivity, and Resting Metabolic Rate in Adults: A Mediator Analysis. Front Endocrinol (Lausanne). 2018 Sep; 9:556.

42 Karampinos DC, Yu H, Shimakawa A, Link TM, Majumdar S. $\mathrm{T}_{1}$-corrected fat quantification using chemical shift-based water/fat separation: application to skeletal muscle. Magn Reson Med. 2011 Nov;66(5):1312-26.

43 Liu CY, McKenzie CA, Yu H, Brittain JH, Reeder SB. Fat quantification with IDEAL gradient echo imaging: correction of bias from T(1) and noise. Magn Reson Med. 2007 Aug;58(2):354-64.

44 Eggers H, Brendel B, Duijndam A, Herigault G. Dual-echo Dixon imaging with flexible choice of echo times. Magn Reson Med. 2011 Jan;65(1):96-107.

45 Ren J, Dimitrov I, Sherry AD, Malloy CR. Composition of adipose tissue and marrow fat in humans by $1 \mathrm{H}$ NMR at 7 Tesla. J Lipid Res. 2008 Sep;49(9):2055-62.

46 Franssens BT, Eikendal AL, Leiner T, van der Graaf Y, Visseren FL, Hoogduin JM. Reliability and agreement of adipose tissue fat fraction measurements with water-fat MRI in patients with manifest cardiovascular disease. NMR Biomed. 2016 Jan;29(1):48-56.

47 Lundström E, Strand R, Johansson L, Bergsten P, Ahlström H, Kullberg J. Magnetic resonance imaging coolingreheating protocol indicates decreased fat fraction via lipid consumption in suspected brown adipose tissue. PLoS One. 2015 Apr;10(4):e0126705.

48 Cypess AM, Lehman S, Williams G, Tal I, Rodman D, Goldfine AB, et al. Identification and importance of brown adipose tissue in adult humans. N Engl J Med. 2009 Apr;360(15):1509-17.

49 Nedergaard J, Bengtsson T, Cannon B. Unexpected evidence for active brown adipose tissue in adult humans. Am J Physiol Endocrinol Metab. 2007 Aug;293(2):E444-52.

50 Skarulis MC, Celi FS, Mueller E, Zemskova M, Malek R, Hugendubler L, et al. Thyroid hormone induced brown adipose tissue and amelioration of diabetes in a patient with extreme insulin resistance. J Clin Endocrinol Metab. 2010 Jan;95(1):256-62.

51 Steinhoff KG, Hankir M, Krause K, Tönjes A, Fenske WK, Sabri O, et al. Interaction of thyroid hormone with brown adipose tissue. Lessons learned from PET-CT. Nucl Med (Stuttg). 2015;54(3):82-7.

52 Weiner J, Hankir M, Heiker JT, Fenske W, Krause K. Thyroid hormones and browning of adipose tissue. Mol Cell Endocrinol. 2017 Dec;458:156-9.

53 Zhang Q, Miao Q, Ye H, Zhang Z, Zuo C, Hua F, et al. The effects of thyroid hormones on brown adipose tissue in humans: a PET-CT study. Diabetes Metab Res Rev. 2014 Sep;30(6):513-20.

54 Nishii R, Nagamachi S, Mizutani Y, Terada T, Kiyohara S, Wakamatsu H, et al. Do TSH, FT3, and FT4 Impact BAT Visualization of Clinical FDG-PET/CT Images? Contrast Media Mol Imaging. 2018 Feb;2018:4898365.

55 Singhal V, Maffazioli GD, Ackerman KE, Lee H, Elia EF, Woolley R, et al. Effect of Chronic Athletic Activity on Brown Fat in Young Women. PLoS One. 2016 May;11(5):e0156353.

56 Obregon MJ. Adipose tissues and thyroid hormones. Front Physiol. 2014 Dec;5:479.

57 Carmona MC, Iglesias R, Obregón MJ, Darlington GJ, Villarroya F, Giralt M. Mitochondrial biogenesis and thyroid status maturation in brown fat require CCAAT/enhancer-binding protein alpha. J Biol Chem. 2002 Jun; 277(24):21489-98.

58 Saito M, Okamatsu-Ogura Y, Matsushita M, Watanabe K, Yoneshiro T, Nio-Kobayashi J, et al. High incidence of metabolically active brown adipose tissue in healthy adult humans: effects of cold exposure and adiposity. Diabetes. 2009 Jul;58(7):1526-31.

59 Brendle C, Werner MK, Schmadl M, la Fougère C, Nikolaou K, Stefan N, et al. Correlation of Brown Adipose Tissue with Other Body Fat Compartments and Patient Characteristics: A Retrospective Analysis in a Large Patient Cohort Using PET/CT. Acad Radiol. 2018 Jan;25(1):102-10.

60 Yoneshiro T, Aita S, Matsushita M, Okamatsu-Ogura Y, Kameya T, Kawai Y, et al. Age-related decrease in coldactivated brown adipose tissue and accumulation of body fat in healthy humans. Obesity (Silver Spring). 2011 Sep;19(9):1755-60.

61 Betz MJ, Enerbäck S. Human Brown Adipose Tissue: What We Have Learned So Far. Diabetes. 2015 Jul;64(7): 2352-60.

62 de Jesus LA, Carvalho SD, Ribeiro MO, Schneider M, Kim SW, Harney JW, et al. The type 2 iodothyronine deiodinase is essential for adaptive thermogenesis in brown adipose tissue. J Clin Invest. 2001 Nov;108(9):1379-85.

63 Wiegratz I, Kutschera E, Lee JH, Moore C, Mellinger U, Winkler UH, et al. Effect of four oral contraceptives on thyroid hormones, adrenal and blood pressure parameters. Contraception. 2003 May;67(5):361-6.

64 Ramey JN, Burrow GN, Polackwich RJ, Donabedian RK. The effect of oral contraceptive steroids on the response of thyroid-stimulating hormone to thyrotropin-releasing hormone. J Clin Endocrinol Metab. 1975 Apr;40(4): 712-4.

65 Hall JA, Ribich S, Christoffolete MA, Simovic G, Correa-Medina M, Patti ME, et al. Absence of thyroid hormone activation during development underlies a permanent defect in adaptive thermogenesis. Endocrinology. 2010 Sep;151(9):4573-82. 